\title{
Experimental investigation of centrifugal fans for personal protection equipment - effect of used 3D printing technologies
}

\author{
Václav Dvořák ${ }^{1, *}$, Radek Votrubec $^{1}$, Jiř Šafka $^{1}$ and Jan Kracík ${ }^{1}$ \\ ${ }^{1}$ Technical University of Liberec, Studentska 2, 46117 Liberec, Czech Republic
}

\begin{abstract}
The aim of the research is experimental investigation of centrifugal fans for a personal protection equipment. The aim of the fan is to drive the contaminated air containing harmful or irritating particles through the filters and then into the mask of workers, such as a fireman, a labourer or a lab worker. The fan is measured on the test stand, the characteristics and performances are evaluated, i.e. the dependencies of the working pressure on the flow rate. The characteristics are measured for three constant speed settings. The characteristics of the wheels produced by the different 3D printing technology are compared. It is found that the production technology has only a minimal effect, the performance of the wheels is more influenced by the position of the impeller on the motor shaft and hence by the mutual position of the impeller and the diffuser.
\end{abstract}

\section{Introduction}

The aim of research is development of new generation of powered air purifying respirators designed to filtrate contaminants in the form of gases, vapours, particles and their combinations. These high performance units guarantee sufficient protection of the wearer even in heavy industrial environments, the chemical industry, laboratories and the pharmaceutical industry [1].

To help people while breathing through filter, a fan is used to propel air flowing in personal respiratory protection systems and units. Because, the reliability and efficiency are crucial for operation of these systems, our work is focused on improvement and optimization of a centrifugal fan used.

Centrifugal fans are used in many applications and therefore, a lot of works can be found. Flow in centrifugal fan is very complex and large number of researchers investigated it both numerically and experimentally.

As Gesner showed, the flow in centrifugal fans is very complicated and complex [2]. Gholamian et al. [3] tested the effect of axial gap between inlet nozzle and impeller on efficiency and flow pattern in centrifugal fans and showed that the minimal axial gap between inlet nozzle and the wheel is not the most favorable.

Even more researchers use CFD [4-6] to calculate flow in centrifugal fans. CFD is also used for optimization of both - backward curved airfoils [7] or the whole centrifugal fan [8].

The aim of the investigation is to verify designed test stand and experimentally investigate high pressure centrifugal fan wheels produced by different technologies.

\section{Experimental investigation}

The section of geometry of the fan wheel is visible in Fig. 1 and Fig. 2. The wheel has a classic design of high pressure fan, i.e. it has twelve long backward curved blades and the width of the wheel decreases, i.e. $b_{2}<b_{1}$, while $d_{1} / d_{2} \approx 0.5$.

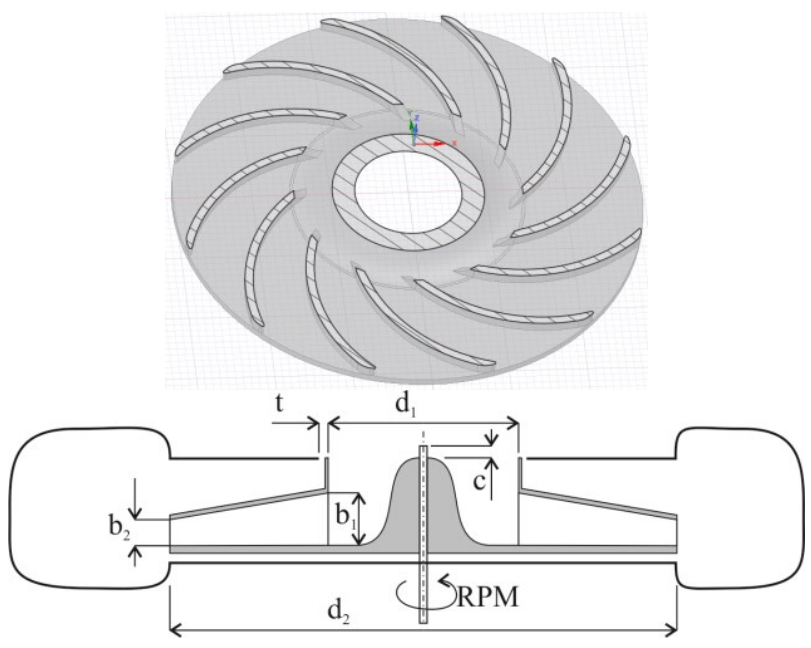

Fig. 1 Design of fan wheel under investigation.

All wheels were mounted in diffuser and engine section, see Fig. 2. It was ensured to keep the axial positon of the wheel on the shaft constant for all variants during investigation, i.e. $c \cong 1.8 \mathrm{~mm}$.

\footnotetext{
* Corresponding author: vaclav.dvorak@tul.cz
} 


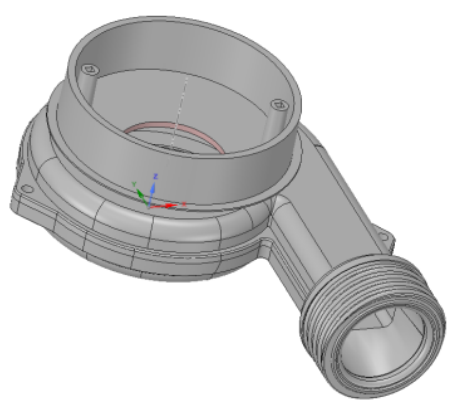

Fig. 2 Design of fan diffuser under investigation.

Eight different wheels manufactured by different technologies were measured. The first wheel (variant 0) was plastic, the rest of wheels (variants $1-7$ ) were made by Rapid prototyping using $3 \mathrm{D}$ printers while using several materials and printing technologies. All wheels are listed in Table 1.

The first technology which we used for 3D printing was PolyJet. It is a photopolymers technology that uses a UV-sensitive liquid photopolymer resin. PolyJet technology works similar like inkjet printing, but instead of jetting drops of ink onto paper, the PolyJet jets layers of sensitive liquid onto a build platform.

This material is cured by thin layers of ultraviolet radiation, see Fig. 3. The size of one layer of the model built in this way is $0.016 \mathrm{~mm}$.

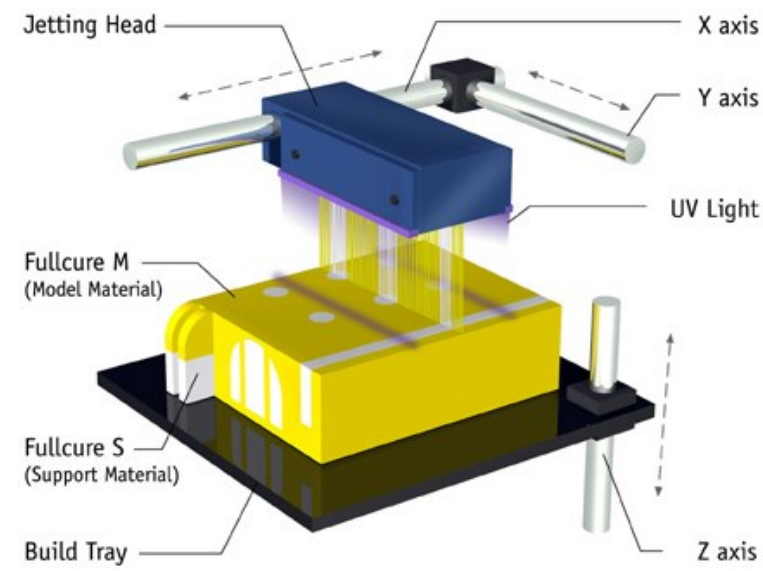

Fig. 3 The principle of PolyJet Matrix method [9]

The second technology of 3D printing was Fused Deposition Modelling (FDM). For this technology material is supplied to a $3 \mathrm{D}$ printer in the form of a fibre on a spool. The fibre is extruded through the print head. The print head moves in the $x y$ plane. When the layer is completed, the head moves in the vertical direction $z$. This technology uses one material to create the geometry of the model and the second material for building the support structures, see Fig. 4. The FDM technology is suitable for solid models without surface quality requirements. It is possible used different thermoplastic polymers: ABS, PLA, PC-ABS, etc.

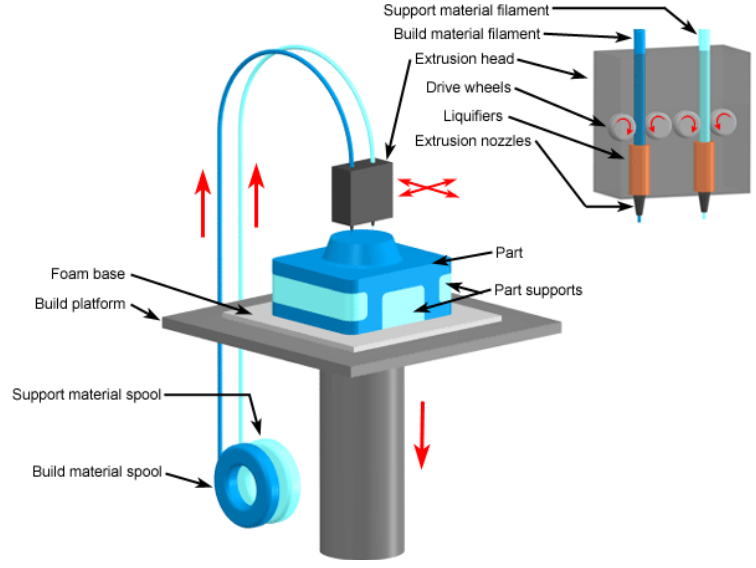

Fig. 4 The principle of FDM technology [10]

As we can see in Table 1, dimensions of printed wheels vary and differ from original plastic wheel. While outer diameters $d_{2}$ of the wheels differ within $0.05 \mathrm{~mm}$, i.e. within $0.1 \%$, the outlet width $b_{2}$ differ within $0.3 \mathrm{~mm}$, i.e. within $8 \%$. Also the inlet width $b_{1}$ of printed wheels was approximately bigger by $0.2 \mathrm{~mm}$, i.e. by $3 \%$.

These differences and also different surface roughness will affect the flow and thus also performance of the fan.

Table 1. Tested wheels, technology, material and dimensions.

\begin{tabular}{|c|l|c|c|}
\hline No. & Technology / material & $\begin{array}{c}\text { 3D print } \\
\text { layer } \\
\text { thickness }\end{array}$ & $d_{2} \times b_{2}$ \\
\hline & & $(\mathrm{mm})$ & $(\mathrm{mm})$ \\
\hline 0 & Injection / plastic & - & $60 \times 3.86$ \\
\hline 1 & PolyJet / VeroClear & 0.014 & $60 \times 4.17$ \\
\hline 2 & PolyJet / VeroBlack & 0.014 & $60.05 \times 4.14$ \\
\hline 3 & PolyJet / VeroGrey & 0.016 & $60.1 \times 4.12$ \\
\hline 4 & PolyJet / ABS-like & 0.03 & $59.95 \times 4.1$ \\
\hline 5 & FDM / ABS-M30 & 0.127 & $59.95 \times 4.21$ \\
\hline 6 & FDM / PC-ABS & 0.127 & $60.05 \times 4.18$ \\
\hline 7 & FDM / ABS- P400 & 0.178 & $60.05 \times 4.02$ \\
\hline
\end{tabular}

The testing stand is visible in Fig. 5. The fan wheel (2) is propelled by electronically controlled brushless DC motor (3). Two different ways were used to control engine output: First, electric current was set to obtain required rotations for low flow resistance. However, it showed that the rotation increased during increasing working pressure. Therefore, the second way was used after that - rotations were specified directly and kept constant during measurements, this way is shown in Fig. 5 . 


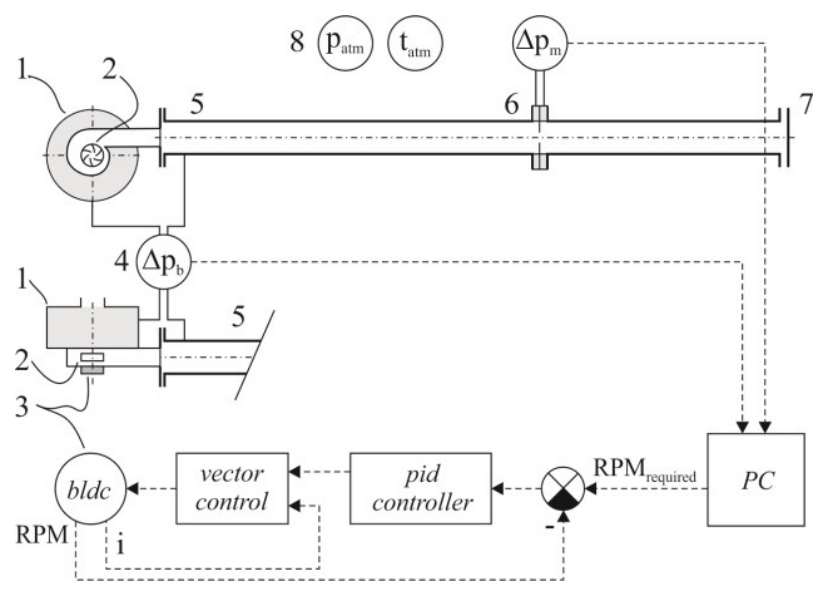

Fig. 5 Testing stand: 1 - suction chamber with filter assembling, 2 - fan wheel, 3 - brushless DC motor, 4 working pressure measuring $\left(\Delta p_{b}\right), 5$ - outflow tube, 6 differential pressure $\Delta p_{m}$ measuring for mass flow specification, 7 - chocking, 8 - measuring of atmospheric pressure and temperature.

The fan was investigated for three values of rotation speed: 6000, 9000 and $11000\left(\mathrm{~min}^{-1}\right)$.

The air is sucked through suction chamber (1), which will later allow assembling of filters, and is compressed and transported into the outflow tube (5). The back pressure and thus the working pressure $\Delta p_{b}$ of the fan is controlled manually by chocking (7) at the tube exit and measured by differential pressure transducer. The mass flow rate is measured by orifice (6), where differential pressure $\Delta p_{m}$ is measured.

The mass flow rate is than specified by relation

$$
\dot{m}=\frac{1}{\sqrt{1-\beta^{4}}} \frac{\pi}{4} d^{2} \varepsilon C \sqrt{2 \Delta p_{m} \rho}
$$

where $\rho$ is air density specified from atmospheric pressure $p_{\text {atm }}$ and temperature $t_{\text {atm }}$ outside the tube, $d$ is orifice diameter, $\beta$ is ratio between orifice and tube diameters, $\varepsilon \cong 1$ is expansion coefficient and $C$ is flow coefficient according standards [11].

\section{Results}

The results of preliminary measurements are carried out in diagram in Fig. 6. There are results only for rotation speed $9000 / \mathrm{min}$ and for two regimes in the diagram: curve A is for constant current and curve B for constant rotation. As we can see, curves differ for low flow rates and higher working pressure. In further works, we used only constant rotation regime, because, if non-constant rotation regime is chosen, final engine rotation can be affected by many factors: flow resistance, the mutual position of the wheel and the shaft, surface roughness of the wheel, etc.

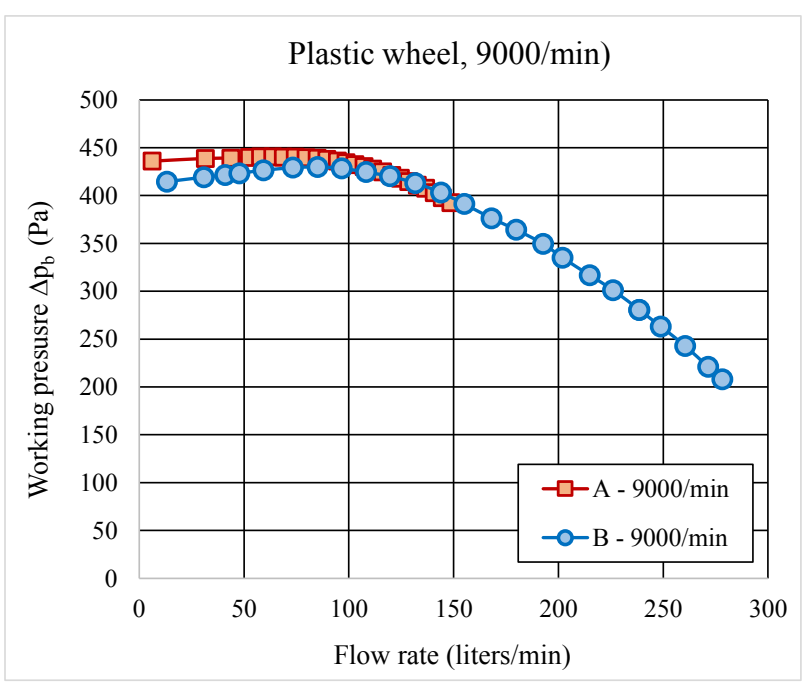

Fig. 6 Fan output for different way of engine control.

The performance curves for plastic wheel, i.e. dependencies of working pressure on flow rate and rotation speed are curried in logarithmic scale in Fig. 7.

It is clear that the fan works according our expectations and that the testing stand is well designed, has good repeatability and reliability. Although, the flow resistant is acceptable for this measuring, it does not allow to measure with filters. Thus, before further investigation, the flow resistance of the test stand should be lowered.

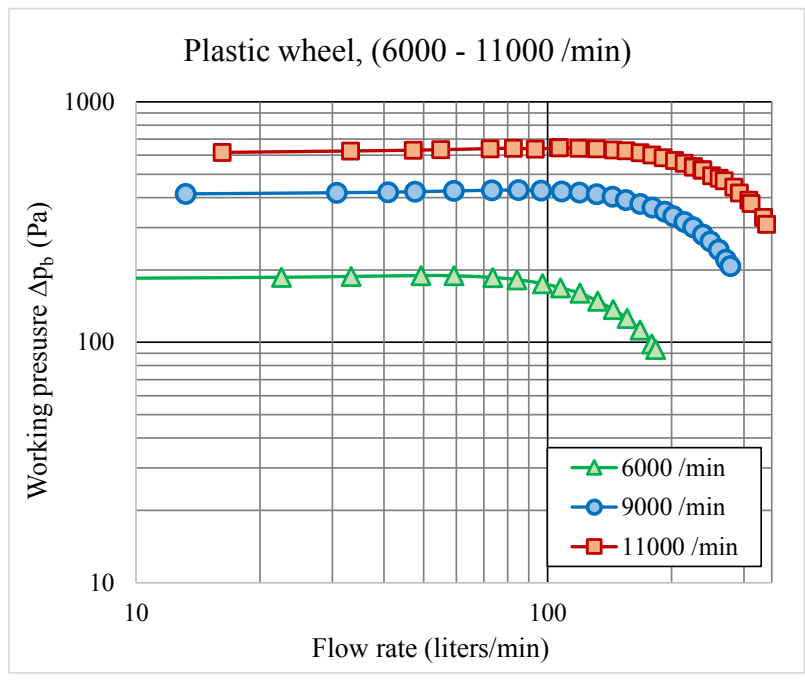

Fig. 7 Fan output for different motor speeds.

The performance curves for all wheels and motor speed $9000 / \mathrm{min}$ are curried in diagram in Fig. 8. Again, we can see that the measuring is very precise with very good repeatability, all curves are quite close to each other. Further, we can obtain that all printed wheels yielded higher power, i.e. higher maximal flow rate and higher maximal working pressure than plastic wheel. We can assume that this difference is connected with bigger outlet width $b_{2}$ of printed wheels, which influences the flow rate proportionally. 


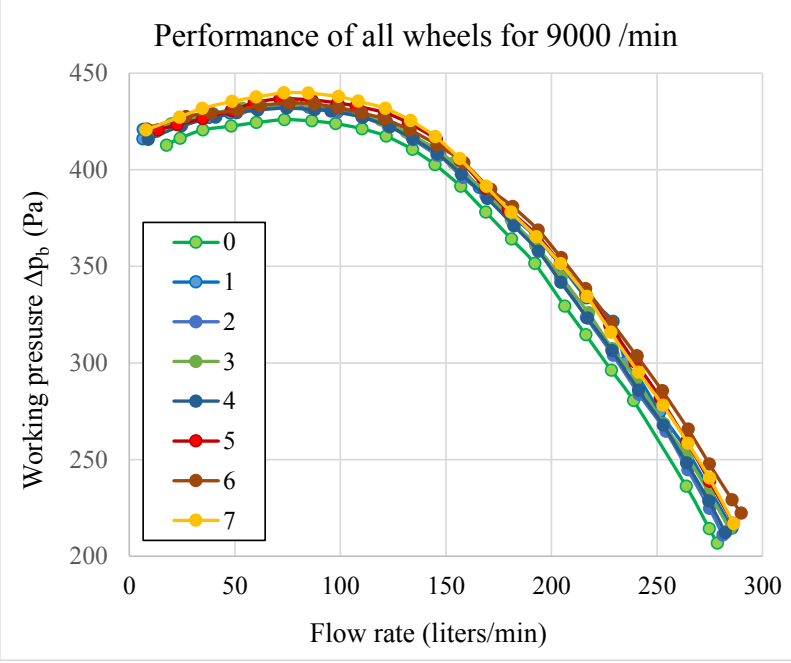

Fig. 8 Fan output for plastic and seven printed wheels for engine speed $9000 / \mathrm{min}$.

Therefore we corrected obtained data according average measured value $b_{2}$, the resulting diagram is in Fig. 9. In this diagram, the maximum flow rate is provided by wheel 7 , the second one is plastic. On the other hand, the plastic wheel still provides the lowest pressure difference for low flow rates.

The highest working pressure provides wheel 7 . According Table 1, this wheel has extremely high roughness which results from high thickness of layers of 3D printing. Also wheels 5 and 6 belong to variants with high performance, while wheels with significantly smoother surfaces, i.e. 1, 2, 3 and 4, generally provide lower flow rates.

We can assume that high roughness of wheel surfaces influences positively power curves by two effects: Firstly, it enables better power transfer from wheel into the fluid, and secondly, it reduces air leak through gap between wheel and fan case around inlet. It seems that reduction of air leak has significant affect for low flow rates.

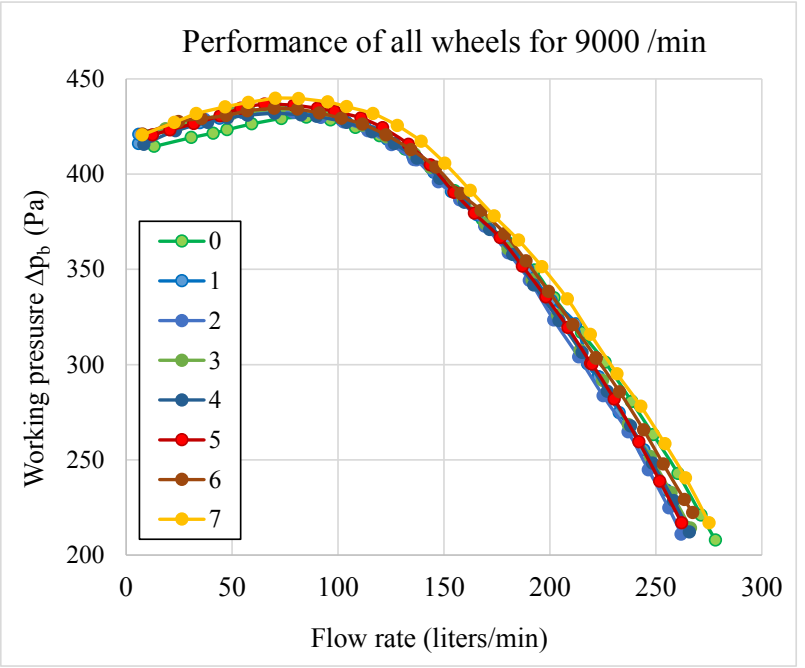

Fig. 9 Fan output for all wheels for motor speed $9000 / \mathrm{min}$ corrected according outlet width $b_{2}$.
Mutual position of wheel and the shaft $c(\mathrm{~mm})$ was another parameter that was investigated. This research, which was performed for wheel no. 7, was done to answer the question of how inaccurate assembling of a wheel on the motor shaft affects its characteristics.

As it is visible in Fig. 10, where low flow rates are presented, the lowest maxima of working pressure is obtained for lowest values of $c$. If $c \geq 1.3 \mathrm{~mm}$, the maximum is approximately the same. We can observe similar situation for high flow rates in Fig. 11. The lowest value of $c$ brings the lowest working pressure.

On the other hand, the situation is opposite for middle values of flow rates: The lower $c$ is, the higher working pressure is achieved. How this can be explained?

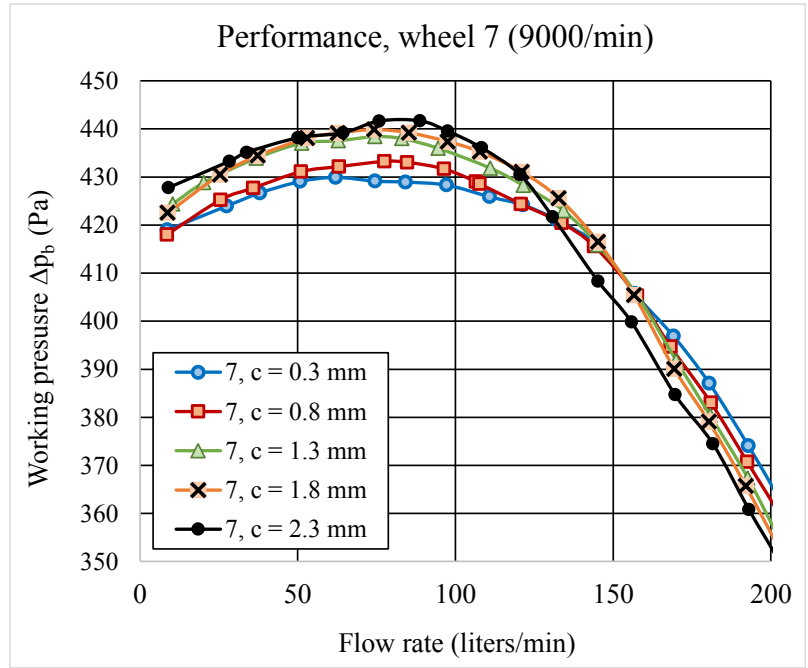

Fig. 10 Fan output for wheel 7 and various mutual position of wheel and the shaft $c$, maximum working pressure.

The answer is following: For low working pressures, there is additional suction into the fan through gab $t$ between the wheel and the diffuser, see Fig. 1. Therefore, low $c$ means low additional suction and lower flow rates for the same pressure. If $c \geq 1.3 \mathrm{~mm}$, the additional suction is restricted by $t$ and these variant shows almost the same data.

For $\Delta p_{b}>270 \mathrm{~Pa}$, see Fig. 11, the additional suction turns into leak and low $c$ becomes an advantage. However, for $\Delta p_{b}>400 \mathrm{~Pa}$ and flow rates $<150$ litres /min, see Fig. 10, another effect becomes significant: The working pressure of a fan is a results of flow inside the wheel. As flow rate decreases, the pressure rise inside the wheel becomes more and more problematic and obtained working pressure decreases. Now, the leak between the rotor and stator allows additional flow through the wheel and thus plays positive role for resulting pressure rise. As a result, the maximal working pressure is obtained for maximal $c$.

Because the standard value of $c$ is $1.8 \mathrm{~mm}$, we can conclude: Until $c$ lies between $1.3 \mathrm{~mm}$ and $1.8 \mathrm{~mm}$, the inaccurate assembling of a wheel on the motor shaft has low or even negligible effect on fan performance. 


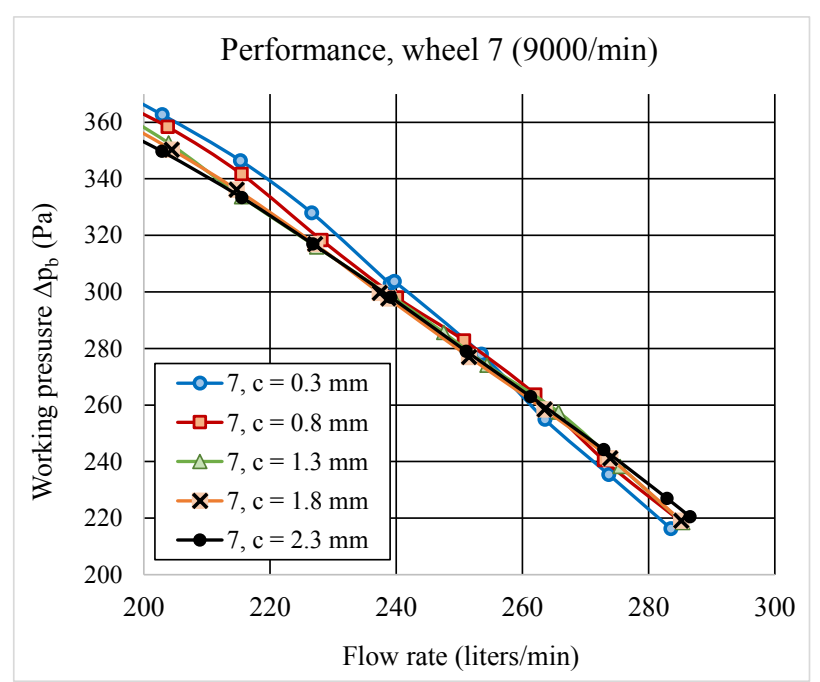

Fig. 11 Fan output for wheel 7 and various mutual position of wheel and the shaft $c$, maximum flow rate.

\section{Conclusions}

One plastic and seven fan wheels printed from various materials and by different technologies were experimentally tested. It was proofed that designed test stand has high accuracy and very good repeatability, even small differences between investigated wheels can be revealed and tested.

The wheels had various dimensions, therefore obtained flow rate was corrected. Obtained data proofed, how inaccurate assembling, i.e. mutual position of a fan wheel and the shaft, can affect fan performance. However, for certain range of position, the influence is negligible.

It follows from measured data that higher surface roughness could be advantageous and bring higher fan performance.

For further research, it would be beneficial to decrease flow resistant of the test stand to allow measuring with assembled filters. Shape and dimension inaccuracies of printed prototypes will be the main problem for further testing and development of new fans. Therefore, it will be necessary and beneficial to engage numerical research too.

\section{Acknowledgement}

This paper was created in the framework of project "Applied research in the field of the new generation of personal protective equipment for the demands of joint rescue service" no. VI20172020052, supported by Ministry of Interior of the Czech Republic.

\section{References}

1. https://www.clean-air.cz

2. F.B. Gessner, ASME Paper no. 67 (1967)

3. M. Gholamian, G. K. M. Rao, B. Panitapu, Case Studies in Therm. Eng. 1, (2013) 26-37

4. J. Zhang, W. Chu, H. Zhang, Y. Wu, X. Dong, Applied Acoustics, 110, (2016) 256-267
5. T. Wang, F. Kong, B. Xia, Y. Bai, C. Wang, Renewable Energy 109 (2017) 518-528

6. S.-C. Lin, M.-L. Tsai, Computers \& Fluids 56 (2012) 24-38

7. M. M. Mohaideen, Procedia Eng. 38 (2012) 15921598

8. B. D. Baloni, Y. Pathak, S.A. Channiwala, Comp. \& Fluids 112 (2015) 72-78

9. http://www.3daddfab.com

10. http://www.custompartnet.com

11. ISO 5167-1:2003 